\title{
Pesquisa sobre vacunación y oportunidades de prevención y educación para la salud. Experiencia en una residencia pediátrica mediante el Mini-Clinical Evaluation Exercise Vaccination status inquiry and hierarchy of prevention and health education opportunities. Experience in a Pediatric Residency using the Mini-Clinical Evaluation Exercise

\author{
Dr. Gonzalo G. Guiñazú ${ }^{a}$ Dr. Nicolás Fortini Cabarcos ${ }^{a}$, Dr. Luis F. Mammi ${ }^{a}$ \\ Dra. Cecilia A. M. Robledo ${ }^{a}$ Dra. María I. Fernández Achával ${ }^{a}$ Dra. Carolina Pascual ${ }^{a}$ \\ y Dra. Christian Elías Costa ${ }^{a}$
}

\section{RESUMEN}

Introducción. ElMini-Clinical Evaluation Exercise (Mini-CEX) es unaherramienta formativa.Dentro de sus ítems, evalúa la pesquisa del estado de vacunación y lajerarquización de oportunidades de prevención.

Objetivos. Determinar porcentaje de pesquisa de dichos ítems en controles de salud y demanda. Comparar desempeño entre primer y tercer año. Materiales y métodos. Análisis retrospectivo de las planillas del Mini-CEX de residentes.

Resultados. Se evaluaron 119 residentes. La prevalencia de pesquisa de vacunación en controles fue $94,9 \%$ (intervalo de confianza-IC-: 91,09-98,91); en demanda, 73,1\% (IC: 65,2-81; OR5,33;IC:2,23-12,75; $\mathrm{p=0,00004).} \mathrm{La} \mathrm{prevalencia}$ de aprovechamiento de oportunidades en controles fue 69,8\% (IC: 61,6-78); en demanda, 62,2\% (IC: 53,5-70,9; OR 1,034; IC: 0,46382,306; $\mathrm{p}=0,4673)$. En 32 residentes se comparó su desempeño entre primer y tercer año en demandas, sin diferencia significativa.

Conclusiones. Se detectó alta prevalencia de pesquisa de vacunación y menor prevalencia de aprovechamiento de oportunidades en controles, con menor pesquisa de ambos ítems en demandas.

Palabras clave: Mini-Clinical Evaluation Exercise,

a. Comité de Docencia e Investigación (CODEI).

Hospital de

Niños Dr. Ricardo

Gutiérrez.

Correspondencia: Dr. Gonzalo Guiñazú: gonzalogermang@gmail. com

\section{Financiamiento: \\ Ninguno.}

Conflicto de intereses: Ninguno que declarar.

Recibido: 20-10-2017 Aceptado: 4-3-2018 opportunities in controls was $69.8 \%$ (CI: 61.678); in demands $62.2 \%$ (CI: 53.5-70.9; OR 1.034; CI: $0.4638-2.306 ; p=0.4673)$. There were 32 trainees compared between their first and third year performance in demands, without significant difference.

Conclusions. We found high prevalence of vaccination inquiry and lower prevalence of hierarchization of prevention opportunities in controls, with less inquiry in demands.

Key words: Mini-Clinical Evaluation Exercise, competency-based education, missed opportunities, vaccination, internship and residency.

http:/ / dx.doi.org/10.5546/ aap.2018.291

Cómo citar: Guiñazú GG, Fortini Cabarcos N, Mammi LF, et al. Pesquisa sobre vacunación y oportunidades de prevención y educación para la salud. Experiencia en una residencia pediátrica mediante el MiniClinical Evaluation Exercise. Arch Argent Pediatr 2018;116(4):291-297.

\section{INTRODUCCIÓN Y OBJETIVOS}

La consulta pediátrica ambulatoria es una instancia de aplicación de herramientas de prevención, educación y promoción de la salud. La promoción se define como el proceso que permite a las personas incrementar el control sobre su salud para mejorarla. ${ }^{1}$ Incluye acciones dirigidas a aumentar las habilidades y capacidades de las personas y a modificar las condiciones que tienen impacto en los determinantes de salud. La educación para la salud comprende oportunidades de aprendizaje y adquisición de habilidades para incrementar el conocimiento de la población y favorecer una mejoría en salud. ${ }^{2}$ 
La vacunación es una de las principales estrategias de prevención primaria. Los esquemas atrasados de vacunación aumentan el riesgo de padecer enfermedades inmunoprevenibles. ${ }^{3}$ Las oportunidades perdidas de vacunación (OPV) y la falta de pesquisa por parte del pediatra constituyen unas de las principales causas de baja cobertura de vacunación en niños. Se considera OPV a cada visita realizada a un centro sanitario por un individuo que precisa ser vacunado $\mathrm{y}$, a pesar de no existir contraindicaciones, no recibe todas las vacunas necesarias. ${ }^{4}$ En nuestro medio, la tasa de prevalencia de OPV es del $19,8 \%,{ }^{5}$ similar a la reportada en la literatura internacional; ${ }^{6}$ el motivo más frecuente es la falta de detección de la necesidad de vacunación. ${ }^{3}$

El Mini-Clinical Evaluation Exercise (Mini-CEX) es una herramienta formativa de evaluación, aplicada durante la observación directa de la práctica clínica asistencial. ${ }^{7}$ Luego de la observación, se mantiene una entrevista con el médico residente para realizar una devolución constructiva. ${ }^{8}$ Este método fue validado paulatinamente a través de su utilización en diferentes programas formativos. ${ }^{9-11}$ En el año 2013, se llevó a cabo un trabajo conjunto entre el Hospital de Niños Dr. Ricardo Gutiérrez y el Hospital Pediátrico "Prof. Dr. Juan P. Garrahan", en el cual un comité asesor de expertos diseñó una planilla de Mini-CEX con la incorporación de una serie de descriptores de la consulta pediátrica agrupados en siete dimensiones (ver Anexo). Estos descriptores fueron validados en las residencias de Clínica Pediátrica de ambos hospitales ${ }^{12}$ y se incorporaron como herramienta de evaluación dentro del programa de residencia.

El Mini-CEX evalúa diferentes competencias relacionadas con la prevención, educación y promoción de la salud en la consulta ambulatoria. Dentro del área de habilidades para la conducción de la entrevista médica, se encuentra el ítem "averiguación del estado de vacunación" y, dentro del área de habilidades para el asesoramiento del paciente y su familia, el ítem "jerarquiza y aprovecha oportunidades de prevención y educación para la salud". ${ }^{6}$

Sobre la base de la experiencia de su utilización, se propuso determinar el porcentaje de pesquisa de los ítems mencionados en atención de controles de salud y demanda espontánea en residentes de primer año. Secundariamente, se buscó comparar el desempeño en dichas áreas entre el primer y el tercer año de su formación.

\section{POBLACIÓN Y MÉTODOS}

Se realizó un análisis retrospectivo sobre la base de las planillas de Mini-CEX de residentes de Clínica Pediátrica del Hospital de Niños Dr. Ricardo Gutiérrez. Se analizaron 4 cohortes de residentes (ingresantes desde 2013 a 2016 inclusive) evaluados en dos instancias durante el primer año de la residencia. La primera observación se realizó en un control de salud y la segunda, en consulta de demanda espontánea en el Servicio de Seguimiento Longitudinal del Niño del Hospital.

Las únicas cohortes evaluadas en una tercera oportunidad fueron las de 2013 y 2014, durante el tercer año de residencia. El ámbito fue una consulta de demanda espontánea en Consultorios Externos. En este grupo, además, se comparó si existieron diferencias de pesquisa de los ítems incluidos entre las consultas de demanda espontánea del primer y tercer año de residencia. Las posibles respuestas a los ítems recolectadas fueron dicotómicas (SÍ/NO). Aquellas respuestas no consignadas en la planilla de evaluación se ingresaron como valor negativo.

Antes del inicio de las evaluaciones, para homogeneizar las observaciones y unificar criterios, se realizaron encuentros de consenso entre los distintos evaluadores. En el primer descriptor, se definió como pesquisa del estado de vacunación el interrogatorio dirigido y/o la constatación del carnet de vacunación del paciente. Con respecto a las oportunidades de prevención y educación, se consensuó que el residente debía interrogar como mínimo sobre planificación familiar, tabaquismo y prevención de accidentes.

Se incluyeron todos los médicos ingresantes a la Residencia de Clínica Pediátrica en el Hospital durante los años 2013-2016 inclusive y a los residentes de tercer año que ingresaron en 2013 y 2014. Se excluyeron aquellos que no completaron dos instancias de evaluación durante el primer año de residencia, una en control de salud y otra en demanda espontánea.

La recolección de datos se realizó utilizando Microsoft Excel ${ }^{\circledR}$. A cada residente se le asignó un código de identificación, que fue utilizado para el ingreso de datos.

En primera instancia, se determinó la prevalencia global de ambos ítems para el total de las consultas. Luego se analizó la diferencia de dichas prevalencias en controles de salud y demandas espontáneas en la población de residentes de primer año. 
Para las cohortes 2013 y 2014, se analizaron las prevalencias de pesquisa por residentes de tercer año en consultas de demanda espontánea. En estas cohortes, se compararon los resultados con los obtenidos durante su primer año de formación.

Se determinaron los intervalos de confianza (IC) para cada variable; se compararon las prevalencias y se calcularon las medidas de efecto y el nivel de significancia de las diferencias halladas. Los cálculos se realizaron con el paquete estadístico STATA $13.0^{\circledR}$. Antes de la recolección de datos, se elaboró un manual estandarizado de procedimientos. En él, se detallaron la codificación de las planillas, el correcto ingreso de datos y cómo consignar aquellos datos ausentes o imprecisos. Se realizó doble entrada de datos, en forma ciega, por dos operadores entrenados. Se compararon los datos ingresados y no se evidenciaron diferencias.

Para la realización de este trabajo, se solicitó la autorización de los jefes de Consultorios Externos y Seguimiento Longitudinal del Niño, del Comité de Ética y del Comité de Docencia e Investigación del Hospital de Niños Dr. Ricardo Gutiérrez. En todos los casos, se requirió el consentimiento de los residentes y se aseguró la confidencialidad de los resultados de las evaluaciones.

\section{RESULTADOS}

Las cohortes de residentes incluidas se muestran en la Tabla 1. Se evaluaron un total de 270 planillas de residentes de primer y tercer año. De estas, 238 correspondieron a 119 residentes de primer año que completaron dos instancias de evaluación y 32 planillas de igual número de residentes de tercer año. En 15 ocasiones, las respuestas no estaban consignadas en la ficha de evaluación, por lo que se ingresaron como valor negativo.

La prevalencia de averiguación del estado de vacunación en el total de las consultas para residentes de primer y tercer año fue $82,6 \%$
(IC: 78,08-87,12; n= 223). La de aprovechamiento de oportunidades de prevención y educación fue 68,15\% (IC: 62,59-73,71; $\mathrm{n}=184$ ).

Para el grupo de residentes de primer año $(n=119)$, se analizaron por separado las planillas de demanda espontánea y control de salud, que completaron un total de 238 planillas. En control de salud, se evidenció una prevalencia de averiguación de estado de vacunación de 94,9\% (IC: 91,09-98,91; $\mathrm{n}=113$ ) y, en demanda espontánea, de 73,1\% (IC: 65,20-81; $\mathrm{n}=87$ ). Considerando un diseño emparejado a partir del test de $\chi_{\text {McNemar }}^{2}$ se obtuvo un OR de 5,33 (IC: 2,23$12,75)$ con una diferencia estadísticamente significativa $(\mathrm{p}=0,00002469)$ (Figura 1). Al evaluar el aprovechamiento de oportunidades de prevención y educación en el control de salud, se observó una prevalencia de $69,8 \%$ (IC: $61,6-78 ; n=83$ ), mientras que, durante la consulta de demanda espontánea, descendió a 62,2\% (IC: 53,51-70,90; $\mathrm{n}=74$ ). El test de $\chi_{\text {McNemar }}^{2}$ evidenció un OR de 1,034 (IC: 0,46-2,31) con una diferencia no significativa $(\mathrm{p}=0,4673)$ (Figura 2).

Como objetivo secundario, para las cohortes 2013 y 2014, se analizaron 32 residentes y se comparó su desempeño entre primer y tercer año en consultas de demanda espontánea. Se observó una prevalencia de "averiguación de estado de vacunación" en primer año de 65,6\% (IC: 50 $82 ; \mathrm{n}=21$ ) $\mathrm{y}$, en tercer año, de $71,8 \%$ (IC: $56,80-$ 86,$80 ; n=23$ ) con un OR de 0,75 (IC: 0,24-2,21; $\mathrm{p}=0,59$ ). Respecto al ítem "aprovechamiento de oportunidades de prevención y educación para la salud", la prevalencia fue, en primer año, de 62,5\% (IC: $45,52-79,54 ; n=20$ ) $y$, en tercer año, aumentó a 84\% (IC: 71-97; n= 27; OR 0,33; IC: 0,06$1,03 ; \mathrm{p}=0,057)$.

\section{DISCUSIÓN}

Desde 1986, se destaca la relevancia de los profesionales de la salud como mediadores de estrategias de promoción, prevención y educación para la salud. ${ }^{1}$ En nuestro ámbito, gran parte de la

TABla 1. Características de las cohortes de residentes

\begin{tabular}{lcccc}
\hline Cohorte & n & Edad en años-media (DE) & Mujeres (\%) & Varones (\%) \\
\hline 2013 & 16 & $25,87(1,43)$ & $15(93,8)$ & $1(6,2)$ \\
2014 & 34 & $26,32(1,33)$ & $31(91,2)$ & $3(8,8)$ \\
2015 & 42 & $26,14(1,18)$ & $37(88,1)$ & $5(11,9)$ \\
2016 & 27 & $26,04(1,19)$ & $23(85,2)$ & $4(14,8)$ \\
Global & 119 & $26,09(1,26)$ & $106(89,1)$ & $13(10,9)$ \\
\hline
\end{tabular}

DE: desvío estándar. 
atención es llevada a cabo por médicos residentes de primer y tercer año en los Consultorios Externos del Hospital. Es debido a ello que su correcto desempeño en la implementación de dichas estrategias adquiere un rol relevante.

En el presente análisis, sobre el total de las consultas estudiadas, se evidenció una alta prevalencia de pesquisa de vacunación en controles de salud. Sin embargo, esta disminuyó significativamente en consultas de demanda espontánea.

La prevalencia global de pesquisa de vacunación coincidió con encuestas reportadas en la bibliografía nacional, cercanas al $86 \% .{ }^{13}$ Además, el porcentaje de aprovechamiento de oportunidades de prevención no alcanzó valores altos en controles de salud ni en consultas de demanda espontánea.
Es interesante destacar el aumento de la prevalencia de todos los ítems pesquisados hacia el tercer año de formación del médico residente. Sin embargo, este aumento no resultó estadísticamente significativo, probablemente, debido a las características de la muestra.

Se estima que la incorporación de las cohortes 2015 y 2016 en un futuro permitirá aumentar la relevancia clínica de estos resultados.

La vacunación es una de las medidas que han tenido mayor impacto en la salud de la población a nivel mundial, ya que es una de las intervenciones más eficientes en salud pública. ${ }^{14,15}$ Sin dejar de jerarquizar la necesidad de mantener altas tasas de pesquisa de estado de vacunación que permitan una adecuada cobertura, se considera importante que los profesionales de la salud adviertan que la vacunación no es la única

FIGURA 1. Porcentaje de pesquisa de estado de vacunación en control de salud vs. demanda espontánea en residentes de primer año

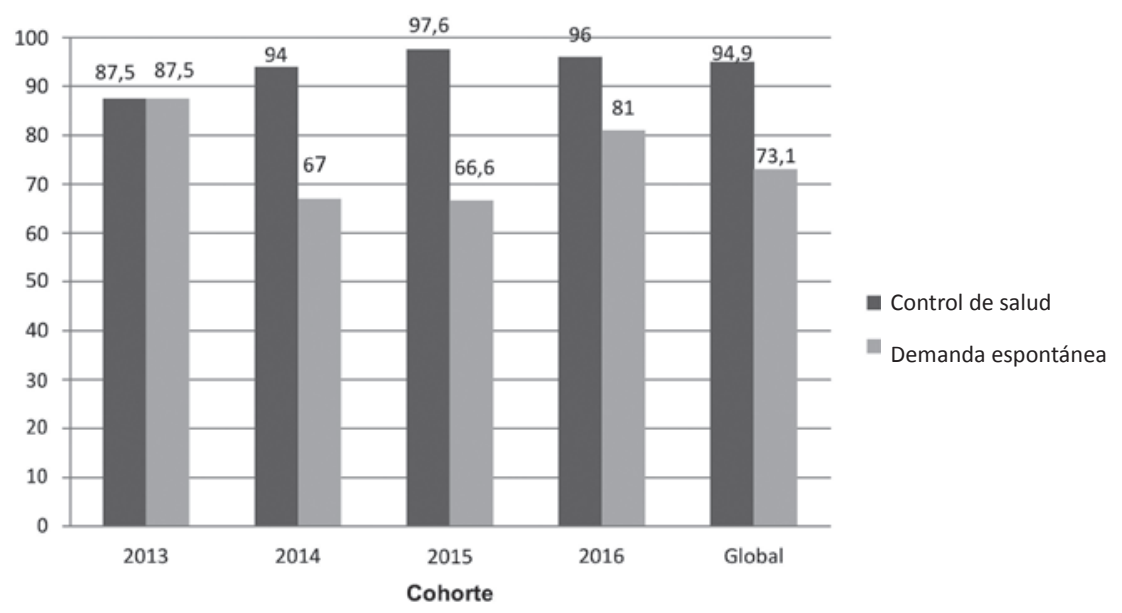

Figura 2. Porcentaje de aprovechamiento de oportunidades de prevención y educación en controles de salud vs. demanda espontánea en residentes de primer año

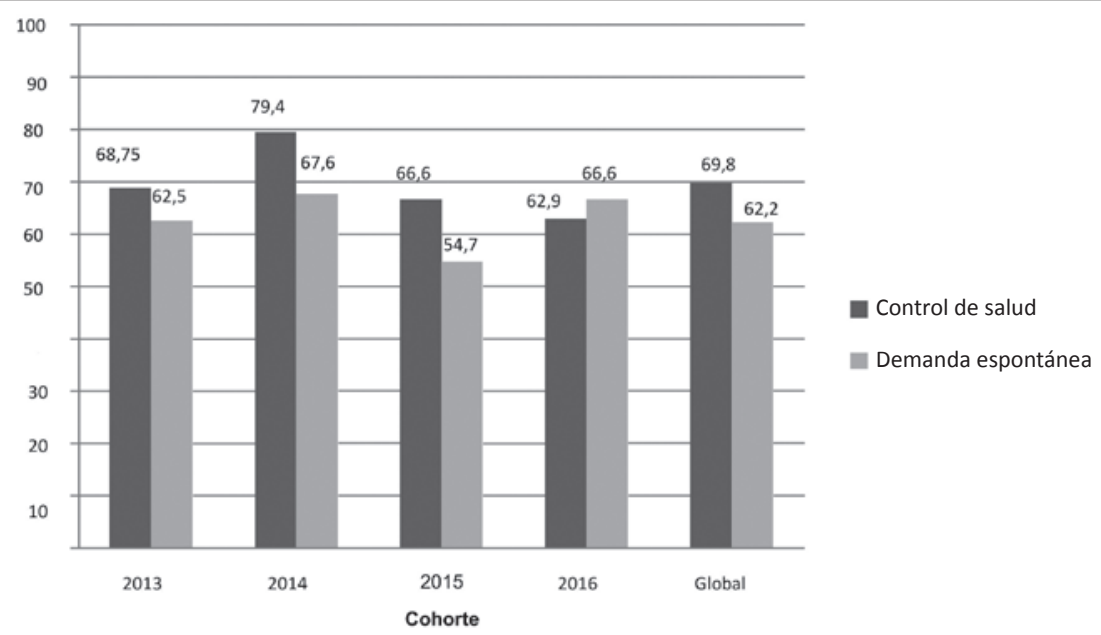


estrategia en salud preventiva, por lo que se deben ponderar otras herramientas de educación y promoción de la salud. Son los sectores más vulnerables de la población los que suelen tener tasas de cobertura de vacunación más bajas, consultan con menor frecuencia y en los que el impacto de medidas de promoción, prevención y educación para la salud en cada acto médico puede ser mayor., ${ }^{4,16,17}$ Es por ello por lo que dichas medidas deberían ser aplicadas tanto en consultas programadas como en demandas espontáneas.

El trabajo tuvo algunas limitaciones. El estudio fue realizado en un único centro asistencial, por lo que la validez externa es limitada. Además, el ítem "jerarquización y aprovechamiento de oportunidades de prevención y educación para la salud" podría resultar difícil de objetivar, ya que distintos evaluadores pudieron haber tenido diferentes criterios para evaluarlo. Para disminuir parcialmente este sesgo, se buscó obtener consenso entre todos los evaluadores, como se mencionó previamente.

Dentro de las fortalezas del estudio, cabe señalar que el Mini-CEX se basa en una observación directa de una consulta médica, lo que permite obtener resultados fácilmente medibles. Esto contrasta con la bibliografía hallada, en la que se analizan datos a partir de encuestas de autopercepción a personal de salud. ${ }^{13}$ Tampoco existe bibliografía previa que analice dichas prevalencias sobre la base de la herramienta Mini-CEX.

\section{CONCLUSIONES}

Las actividades de promoción de la salud y prevención primaria constituyen uno de los ejes principales de la consulta pediátrica. En la población analizada, se detectó una alta prevalencia de averiguación de estado de vacunación y menor prevalencia de aprovechamiento de oportunidades de prevención en controles programados. Además, se evidenció una menor pesquisa de ambos ítems en consultas de demanda espontánea. Secundariamente, se constató un mayor porcentaje de pesquisa en residentes de tercer año al comparar con su desempeño en primer año, aunque esta diferencia no resultó estadísticamente significativa.

\section{Agradecimientos}

Al Dr. Nicolás Falk, médico de planta de Consultorios Externos de Pediatría, Hospital de Niños Dr. Ricardo Gutiérrez.

\section{REFERENCIAS}

1. World Health Organization. The Ottawa Charter for Health Promotion. First International Conference on Health Promotion, Ottawa, 21 November 1986. [Acceso: 18 de agosto de 2017]. Disponible en: http:/ / www.who. int/healthpromotion/ conferences / previous / ottawa/en / index1.html.

2. World Health Organization. Promoción de la salud. Glosario. Ginebra:Organización Mundial dela Salud; 1998. [Acceso:18 de agosto de 2017]. Disponibleen: http:/ / apps. who.int/iris/handle/10665/67246.

3. Spagnuolo de Gentile A, Rearte A, Regatky N, et al. Esquemas atrasados y oportunidades perdidas de vacunación en niños de hasta 2 años atendidos en centros de salud. Rev Argent Salud Pública 2012; 3(11):30-6.

4. ÚbedaSansanoMI.Oportunidades perdidas de vacunación. Rev Pediatr Aten Primaria 2005; 7(Supl 4):S43-54.

5. Gentile A, Bakir J, Firpo V, et al. Esquemas atrasados de vacunación y oportunidades perdidas de vacunación en niños de hasta 24 meses: estudio multicéntrico. Arch Argent Pediatr 2011; 109(3):219-25.

6. Luman ET, McCauley MM, Stokley S, et al. Timeliness of childhood immunizations. Pediatrics 2002; 110(5):935-9.

7. American Board of Internal Medicine. The Mini-CEX: A quality tool in evaluation. Guidelines and implementation strategies from program directors. [Acceso: 18 de agosto de 2017]. Disponible en: http: / / www.personalbesthealth. com/Literature\%20for $\% 20$ Web / Articles / Mini-CEX\%20 Guidelines.pdf.

8. Fornells-Vallés JM. El ABC del Mini-CEX. Educ Med 2009; 12(2):83-9.

9. Durning SJ, Cation LJ, Markert RJ, et al. Assessing the reliability and validity of the mini-clinical evaluation exercise for internal medicine residency training. Acad Med 2002; 77(9):900-4.

10. Alves de Lima A, Henguin R, Thierer J, et al. A qualitative study of the impact on learning of the mini clinical evaluation exercise in postgraduate training. Med Teach 2005; 27(1):46-52.

11. Alves de Lima A, Van der Vleuten C. Efecto educativo de la evaluación del desempeño profesional: el caso del miniCEX. Medicina (B Aires) 2013; 73(3):286-7.

12. Abadie Y, Battolla J, Zubieta A, et al. Uso de descriptores durante la implementación de Mini-CEX en la Residencia de Pediatría. Medicina (B Aires) 2015; 75(5):289-96.

13. Federicci MI, Gatica CI, Nalda G, et al. Oportunidades perdidas de vacunación. Participación del personal de salud. Arch Argent Pediatr 1999; 97(1):3-7.

14. Gentile A, Juárez M, Hernández S, et al. Influenza vaccine: Delayed vaccination schedules and missed opportunities in children under 2 years old. Vaccine 2015; 33(32):3913-7.

15. World Health Organizaton. Department of Immunization, Vaccines and Biologicals and UNICEF ProgrammeDivision, Health section. GIVS global immunization vision and strategy 2006-2015. October 2005. [Acceso: 13 de octubre de 2017]. Disponibleen: http:/ / whqlibdoc.who.int/hq/2005/ WHO_IVB_05.05.pdf.

16. Szilagyi PG, Rodewald LE. Missed opportunities for immunizations: a review of the evidence. J Public Health Manag Pract 1996; 2(1):18-25.

17. Kempe A, Lowery NE, Pearson KA, et al. Immunization recall: effectiveness and barriers to success in an urban teaching clinic. J Pediatr 2001; 139(5):630-5. 
ANEXO

PLANILLA DEL MINI-CLINICAL EVALUATION EXERCISE PEDIÁTRICO EN FORMATO PAPEL

Residente:

Año:

Fecha:

ID del observador:

Problema del paciente/diagnóstico:

Internación por 1.a vez. Internación de seguimiento. Ambulatorio (control de salud). Ambulatorio (demanda espontánea).

Baja complejidad. Mediana complejidad. Alta complejidad.

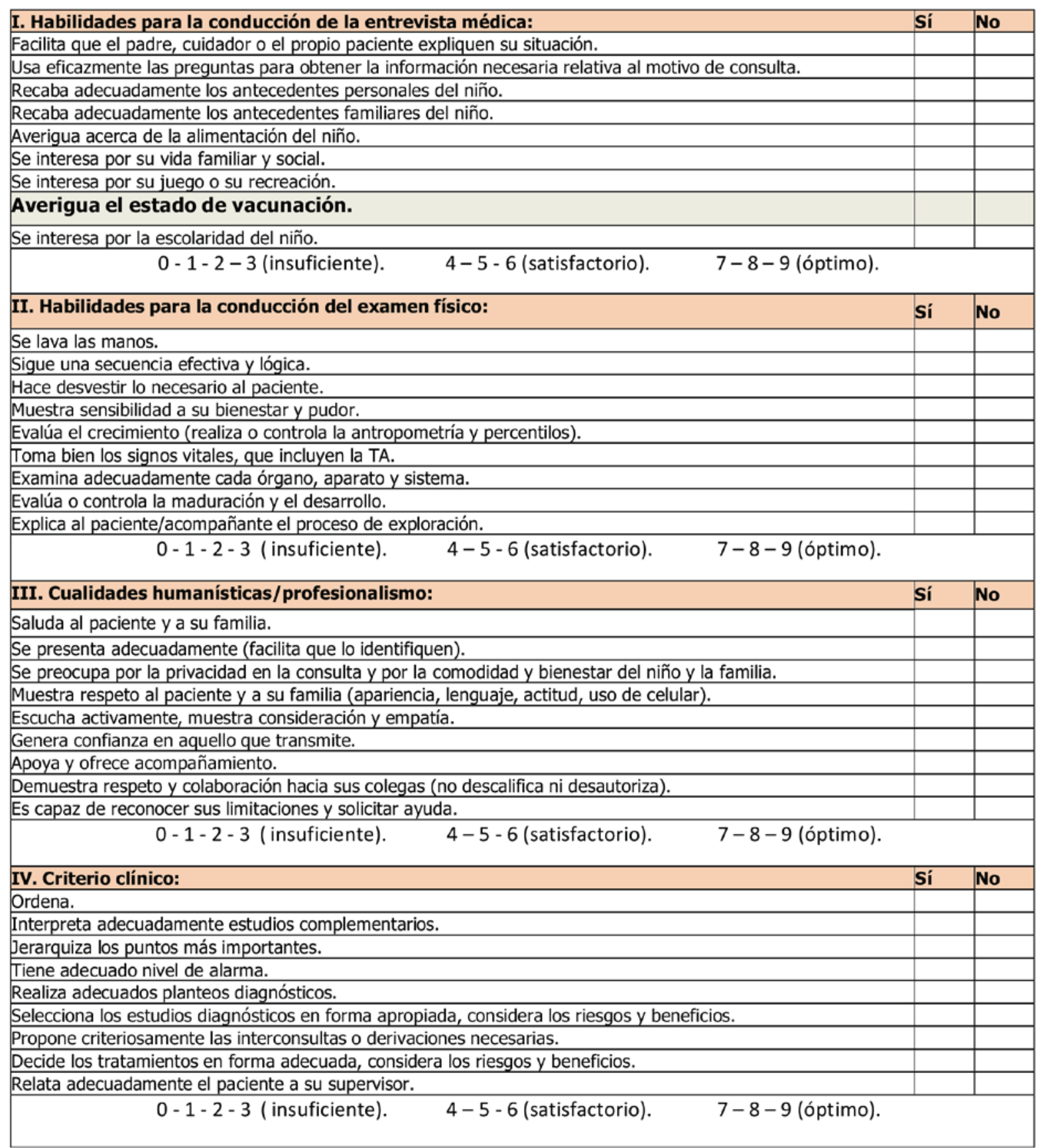




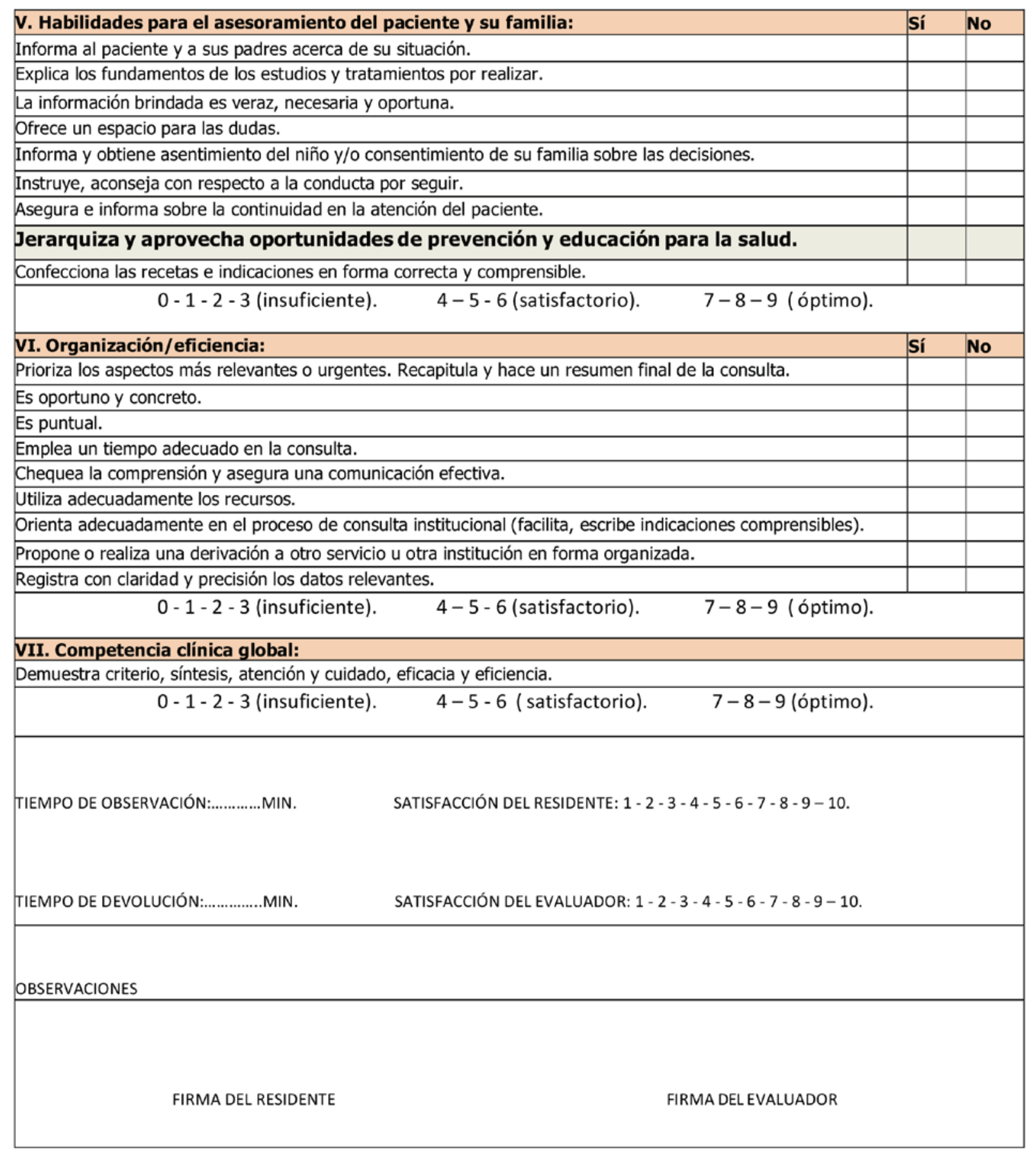

